\title{
Multiple choice questions: ANSWERS
}

Chapter 1. An overview of valvular heart disease in the elderly

What is the most frequent valvulopathy in the elderly?

a. Aortic stenosis

2. To date, what are the indications for transcatheter aortic valve replacement?

Contraindicated people for conventional surgery

High-risk people for conventional surgery

3. What are the main surgical difficulties to consider for mitral valve surgery in the elderly?

Mitral annular calcifications

Required associated procedures increasing the operative risk What are the primary goals of valvular surgery in the elderly?

To increase the life expectancy

To provide a better quality of life

To allow another required surgery

5. What are the main postoperative complications in such patients?

a. Postoperative encephalopathy

b. Renal failure

c. Lung dysfunction

Anemia

\section{Chapter 2. Molecular mechanisms of calcific aortic valve disease}

Select the best answer. Inflammation in calcific aortic valve disease (CAVD) is:

All of the above 
2. Regarding the lipid retention process in CAVD, what is the best answer?

Biglycan is elevated in stenotic aortic valve and participates to the retention of lipid and inflammation

3. Select the best statement:

Ectonucleotidases are involved in the mineralization of the aortic valves

Select the best answer on the role of the renin angiotensin system in calcific aortic valve disease:

Activation of the renin-angiotensin system is associated with aortic valve inflammation

Select the false statement:

Sirtuins are not involved in the control of resistin expression in stenotic aortic valves

\section{Chapter 3. Assessing operative risk in elderly patients with heart valve disease}

Which is the most prevalent etiology of valve disease in the elderly?

Degenerative

2. Which is the prevalence of frailty in the elderly population?

a. Up to $50 \%$

In which multicenter, randomized trial was a frailty index used to define patients' inoperability?

PARTNER

Which is the prevalvence of aortic stenosis in patients over 75 years of age according to Euro Heart Survey data?

$56 \%$

5. Which of the following is not a frailty parameter?

Active smoker

Chapter 4. Transcatheter aortic valve replacement in the high-risk elderly

There are a lot of imaging modalities when considering a patient for a transcatheter aortic valve replacement (TAVR). Which 
imaging modality provide the most accurate information about the aortic annulus size and shape?

Multimodality cardiac imaging including echocardiography, angiography and multidetector computed tomography

2. What are the main characteristics that you take into account when you consider referring a patient to TAVR?

Besides the risk scores, functional status and existing comorbidities such as porcelain aorta, previous radiation therapy to the chest, cirrhosis and general frailty

What are the approaches used for TAVR?

Antegrade approach, transapical approach and retrograde approach via the femoral artery, axillary artery, subclavian artery and direct puncture of the ascending aorta

TAVR is appropriate for only a highly selected population and patient selection requires the multidiciplinary heart team. Who are the physicians that should be in the heart team?

Cardiologists, cardiothoracic surgeons and specialists in cardiovascular imaging

5. What are the main points that provide the success of TAVR?

All of the above

\section{Chapter 5. Aortic stenosis}

Select the best statement.

All of the above statements are correct

2. Select the best statement.

Aortic sclerosis is found in more than $35 \%$ of patients aged over 75 years

Select the best statement.

All of the above statements are correct

Select the best statement.

Symptomatic, severe AS is considered to be a definite surgical indication

Select the best statement.

All of the above statements are correct 


\section{Chapter 6. Management and follow-up of valvular disease in the geriatric population}

Rank syncope, congestive heart failure and angina in the worst order of prognosis?

Congestive heart failure, syncope, angina

2. Are bioprostheses or mechanical prostheses the best choice for the elderly for management of severe aortic stenosis?

Bioprostheses

When counseling patients is it best to consider actuarial or actual methodology in the support of prostheses type?

Actual (cumulative incidence)

Are risk scores better than evaluation of disability and frailty in determining candidates for TAVI?

Disability and frailty

TAVI indications should be 'on label' or 'off label'?

On label

Is valve-in-valve for structural valve deterioration of previous bioprostheses in the aortic position best performed for valve size $<23$ or $\geq 23 \mathrm{~mm}$ ?

$\geq 23 \mathrm{~mm}$

Is stroke rate higher after SAVR or TAVI?

TAVI

Is echocardiographic surveillance best performed on a routine schedule or only if symptoms warrant the investigation?

Both routine basis and if symptoms occur 